\title{
Non-Abelian chiral spin liquid on a simple non-Archimedean lattice
}

Peri, V ; Ok, S ; Tsirkin, S S ; Neupert, T ; Baskaran, G ; Greiter, M ; Moessner, R ; Thomale, R

\begin{abstract}
We extend the scope of Kitaev spin liquids to non-Archimedean lattices. For the pentaheptite lattice, which results from the proliferation of Stone-Wales defects on the honeycomb lattice, we find an exactly solvable non-Abelian chiral spin liquid with spontaneous time-reversal symmetry breaking due to lattice loops of odd length. Our findings call for potential extensions of exact results for Kitaev models which are based on reflection positivity, which is not fulfilled by the pentaheptite lattice. We further elaborate on potential realizations of our chiral spin-liquid proposal in strained $-\mathrm{RuCl} 3$.
\end{abstract}

DOI: https://doi.org/10.1103/physrevb.101.041114

Posted at the Zurich Open Repository and Archive, University of Zurich

ZORA URL: https://doi.org/10.5167/uzh-186471

Journal Article

Published Version

Originally published at:

Peri, V; Ok, S; Tsirkin, S S; Neupert, T; Baskaran, G; Greiter, M; Moessner, R; Thomale, R (2020). Non-Abelian chiral spin liquid on a simple non-Archimedean lattice. Physical review B, 101(4):041114.

DOI: https://doi.org/10.1103/physrevb.101.041114 


\title{
Non-Abelian chiral spin liquid on a simple non-Archimedean lattice
}

\author{
V. Peri $\odot,{ }^{1}$ S. Ok, ${ }^{2}$ S. S. Tsirkin $\odot,{ }^{2}$ T. Neupert $\odot,{ }^{2}$ G. Baskaran,,${ }^{3,4}$ M. Greiter $\odot,{ }^{5}$ R. Moessner, ${ }^{6}$ and R. Thomale $\odot^{5, *}$ \\ ${ }^{1}$ Institute for Theoretical Physics, ETH Zurich, CH-8093 Zurich, Switzerland \\ ${ }^{2}$ Department of Physics, University of Zurich, Winterthurerstrasse 190, CH-8057 Zurich, Switzerland \\ ${ }^{3}$ Institute of Mathematical Sciences, Chennai 600 113, India \\ ${ }^{4}$ Perimeter Institute for Theoretical Physics, Waterloo, Ontario, Canada N2L $2 Y 5$ \\ ${ }^{5}$ Institute for Theoretical Physics and Astrophysics, University of Würzburg, Am Hubland, D-97074 Würzburg, Germany \\ ${ }^{6}$ Max-Planck-Institut für Physik komplexer Systeme, Nöthnitzer Strasse 38, D-01187 Dresden, Germany
}

(Received 14 August 2019; revised manuscript received 28 November 2019; published 30 January 2020)

\begin{abstract}
We extend the scope of Kitaev spin liquids to non-Archimedean lattices. For the pentaheptite lattice, which results from the proliferation of Stone-Wales defects on the honeycomb lattice, we find an exactly solvable non-Abelian chiral spin liquid with spontaneous time-reversal symmetry breaking due to lattice loops of odd length. Our findings call for potential extensions of exact results for Kitaev models which are based on reflection positivity, which is not fulfilled by the pentaheptite lattice. We further elaborate on potential realizations of our chiral spin-liquid proposal in strained $\alpha-\mathrm{RuCl}_{3}$.
\end{abstract}

DOI: 10.1103/PhysRevB.101.041114

Introduction. Since the first proposal [1], quantum spin liquids have remained an as fascinating as elusive direction of contemporary condensed matter research on frustrated magnetism and topologically ordered many-body states. In theory, different approaches have been developed, many of which were inspired by cuprate superconductors [2] or the fractional quantum Hall effect (FQHE) [3], but these were limited due to the relative paucity of exactly solvable models [4,5]. A fundamental breakthrough was reached by Kitaev in proposing a microscopic Hamiltonian for quantum spin liquids with an emergent massive Ising gauge theory [6]. Instead of just realizing a desired spin-liquid ground-state wave function as an exact eigenstate of a microscopic Hamiltonian, the powerful exact solution of the Kitaev spin liquid allows for the explicit analysis of anyonic excitations. Its solution is most elegantly accomplished by a Majorana representation, where the eigenspectrum simplifies to a free single-Majorana band structure.

The Kitaev models realize both Abelian and non-Abelian anyons [6], spontaneous time-reversal symmetry-breaking chiral spin liquids $[7,8]$, a generalization to $\mathbb{Z}_{k}$ gauge theory [9], and an extension to three-dimensional spin liquids with anyon metallicity [10-12]. While the non-Abelian anyons in the Kitaev model are of Ising type, alternative microscopic approaches to non-Abelian spin liquids have found realizations of SU(2) $k$ anyons in chiral [13-17] and nonchiral [18] spin liquids. The concept of spinon Fermi surfaces has been previously developed in the context of Gutzwiller projections on fermionic mean-field states [19]. The exact solvability of the Kitaev models, however, renders all these features accessible to an unprecedented degree, and as such promises a more concise connection to observable quantities [20] and candidate materials [21-25].

*rthomale@physik.uni-wuerzburg.de
In this Rapid Communication, we extend the Kitaev paradigm to non-Archimedean lattices. Lattices can be classified by the symmetry of sites and bonds. Archimedean lattices are formed by regular polygons where each lattice vertex is surrounded by the same sequence of polygons. This implies the equivalence of all sites, but not necessarily of all bonds. Conversely, for lattices of the type including the Lieb lattice [26], the symmetry equivalence of all bonds does not imply the equivalence of sites. In a non-Archimedean lattice, neither all sites nor all bonds are equivalent. As a prototypical example to which we particularize in the following, pentaheptite [Fig. 1(a)] exhibits irregular pentagons and heptagons as well as two types of vertices with $\left(5^{1}, 7^{2}\right)$ and $\left(5^{2}, 7^{1}\right)$ configurations, respectively. In this notation $a^{m}$, the lattice is characterized by a list of the number of edges $a$ and the multiplicity $m$ of polygons that surround each inequivalent vertex. Pentaheptite $[27,28]$ can be thought of as originating from the honeycomb $\left(6^{3}\right)$ lattice by the proliferation of Stone-Wales defects. There, a pair of honeycomb bonded vertices change their connectivity as they rotate by $90^{\circ}$ with respect to the midpoint of their bond. The Stone-Wales defect proliferation transforms four contingent hexagons into two heptagons and two pentagons. Pentaheptite has three-colorable bonds as the honeycomb lattice, and thus lends itself to an exact solution of the Kitaev model, albeit not being three-colorable by faces.

Strain engineering of pentaheptite lattice in $\alpha-\mathrm{RuCl}_{3}$. We perform first-principles calculations of the candidate Kitaev honeycomb material $\alpha-\mathrm{RuCl}_{3}$ under uniaxial strain (see Supplemental Material [29] and Refs. [30-34] therein for more details). We find that under sufficiently strong tensile or compressive strain a configuration where the $\mathrm{Ru}$ atoms arrange themselves in a pentaheptite lattice becomes favorable [see Fig. 1(d)]. This result motivates our choice to extend the exactly solvable Kitaev model to non-Archimedean lattices. Moderate strain in $\alpha-\mathrm{RuCl}_{3}$ has been shown to enhance 

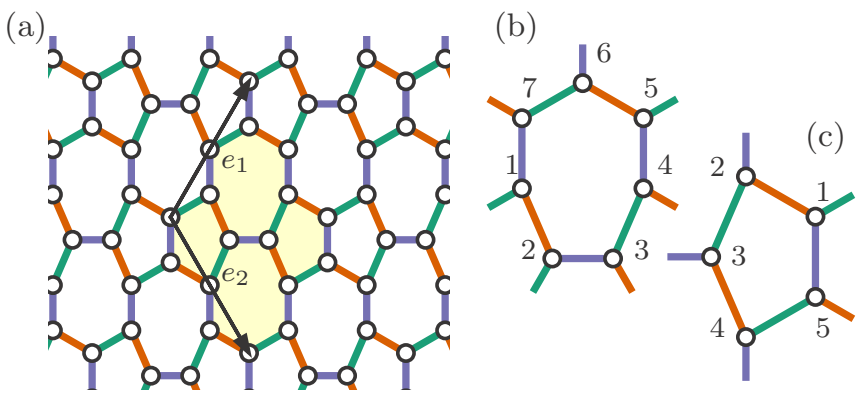

(d)

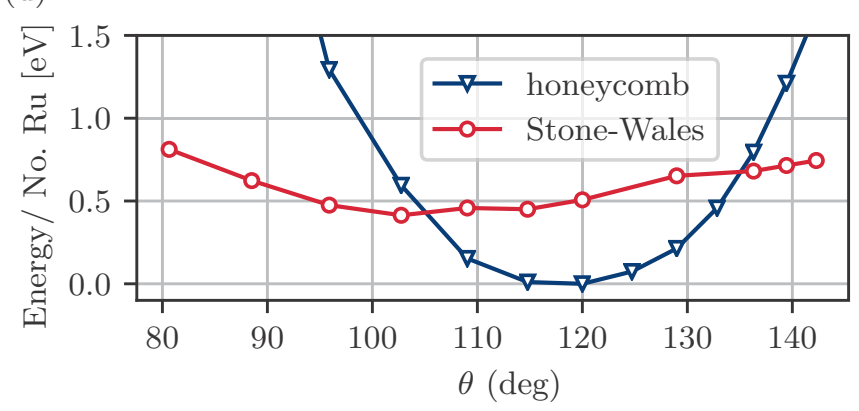

FIG. 1. (a) Pentaheptite lattice with a unit cell highlighted in light yellow and the lattice vectors $\boldsymbol{e}_{1}$ and $\boldsymbol{e}_{2}$. Bond colors highlight the type of spin-spin coupling across a bond $\sigma_{j}^{\alpha} \sigma_{k}^{\alpha}, \alpha=x, y, z$ (violet for $z$, orange for $y$, and green for $x$ ). (b), (c) Site labels for the definition of plaquette operators in (2). (d) Energy per Ru atoms for $\alpha-\mathrm{RuCl}_{3}$ under strain with respect to the energy of the honeycomb configuration at $\theta=120^{\circ}$. In red for a honeycomb lattice, in blue for a Stone-Wales defect. The strain changes the angle $\theta$ between the lattice vectors and the Stone-Wales structure is preferred at $\theta<105^{\circ}$ and $\theta>135^{\circ}$.

magnetic Kitaev interactions [35]. It remains an open question whether the geometric frustration introduced by stronger strain [29] is detrimental to the directional Kitaev exchange.

Kitaev pentaheptite model. We consider a spin-1/2 degree of freedom on each pentaheptite site. The unit cell shown in Fig. 1(a) contains eight sites and is spanned by the vectors $\boldsymbol{e}_{1}=(\sqrt{3} a, 3 a)$ and $\boldsymbol{e}_{2}=(\sqrt{3} a,-3 a)$. We set the nearestneighbor distance $a$ of the underlying honeycomb lattice to unity. The Kitaev Hamiltonian reads

$$
H=-J_{x} \sum_{x \text {-type }} \sigma_{j}^{x} \sigma_{k}^{x}-J_{y} \sum_{y \text {-type }} \sigma_{j}^{y} \sigma_{k}^{y}-J_{z} \sum_{z \text {-type }} \sigma_{j}^{z} \sigma_{k}^{z},
$$

where $\sigma_{j}^{x, y, z}$ denotes the Pauli matrices acting on site $j$, the sums run over distinct sets of bonds connecting nearestneighbor sites $j$ and $k$, and $J_{x, y, z} \in \mathbb{R}$. The bonds which contribute to each sum are shown in Fig. 1(a). Each heptagon and pentagon of the lattice is associated with a conserved quantity of (1) given by

$$
\begin{aligned}
& W_{\text {pen }}=K_{12} K_{23} K_{34} K_{45} K_{51}, \\
& W_{\text {hep }}=K_{12} K_{23} K_{34} K_{45} K_{56} K_{67} K_{71},
\end{aligned}
$$

where $K_{i j}=\sigma_{i}^{\alpha} \sigma_{j}^{\alpha}$ for sites $i$ and $j$ connected by a bond of type $\alpha=x, y, z$. The spin operators act on the sites around each pentagon and heptagon according to the site labels in Figs. 1(b) and 1(c), respectively. The conserved quantities for the pentagons and heptagons that relate to those shown in Figs. 1(b) and 1(c) via mirror reflection are defined analogously. All $W_{\text {pen }}$ and $W_{\text {hep }}$ commute with each other and the Hamiltonian (1), which can thus be diagonalized in each eigenspace of these operators ("flux sector") separately.

Importantly, in contrast to the Kitaev honeycomb case, time-reversal $T$ commutes with the plaquette operators $W_{l}$ but flips their eigenvalues. Applying $T$ to the equation $W_{l}|\psi\rangle=$ $w_{l}|\psi\rangle$, one gets $W_{l} T|\psi\rangle=w_{l}^{*} T|\psi\rangle$. The reason is that the elementary loops in the pentaheptite lattice are of odd length and have imaginary eigenvalues $\pm i$. This implies spontaneous time-reversal symmetry breaking. In particular, one needs to specify the direction followed around the plaquette and in definition (2) we choose a counterclockwise convention. A similar situation is found on the decorated honeycomb lattice $\left(3,12^{2}\right)$ of the Kitaev-Yao-Kivelson (KYK) model [7]. In our case, however, all elementary loops are of odd length.

With the conserved plaquette quantities identified, one can map the system to noninteracting Majorana fermions in each flux sector by following Kitaev's procedure [6]: We replace each spin (site $j$ ) by four Majorana fermions $c_{j}, b_{j}^{\alpha}, \alpha=$ $x, y, z$, and restrict the Hilbert space to that of even fermion parity on each site [36]. The resulting Hamiltonian takes the form

$$
H=\frac{i}{4} \sum_{\langle j k\rangle} A_{j k} c_{j} c_{k},
$$

where the sum runs over nearest-neighbor sites and $A_{j k}=$ $2 J_{\alpha_{j k}} u_{j k}$ if sites $j$ and $k$ are connected by an $\alpha$ link, $\alpha_{j, k} \in$ $\{x, y, z\}$. The Majorana bilinears $u_{j k}=i b_{j}^{\alpha_{j k}} b_{k}^{\alpha_{j k}}$ commute with each other and the Hamiltonian. As Hermitian operators that square to 1 , we can replace them by their eigenvalues \pm 1 . Their eigenvalues are related to those of $W_{\text {pen }}$ via

$$
W_{\text {pen }}=(-i)^{5} \prod_{\langle j k\rangle \in \text { pen }} u_{j k}
$$

where the product runs over all bonds that form a given pentagon. The analogous equation holds for $W_{\text {hep }}$ with the prefactor $(-i)^{7}$. Thus, while the eigenvalue of $u_{j k}$ on a given bond is gauge variant, the product of eigenvalues around a closed loop is a gauge invariant $\mathbb{Z}_{2}$ flux. Note that the order in the product of Eq. (4) requires one to fix a positive direction for the bond operators $u_{j k}$ [29]. According to our convention, the configuration with all the $u_{i j}$ with positive eigenvalues corresponds to heptagonal (pentagonal) plaquettes with eigenvalue $+i(-i)$.

Identifying the ground-state flux sector. For each flux sector, the ground-state energy of Eq. (3) can be determined. A powerful result by Lieb [37], based on reflection positivity, assures that if a Kitaev-type spin model possesses reflection symmetry such that the plane of reflection does not contain any lattice site, a ground state is always in the flux-free sector. The Kitaev model on the pentaheptite lattice is particular in that it does not have such a mirror symmetry.

From our flux sector analysis, we conjecture that even for Eq. (1), the ground state is in the flux-free sector, i.e., the sector where all $u_{i j}$ have positive eigenvalues according to the chosen convention. Numerical evidence along this line has been provided for other systems lacking reflection positivity, while a rigorous result is missing [38]. Fixing $J_{x}=J_{y}=J_{z}=1$ 


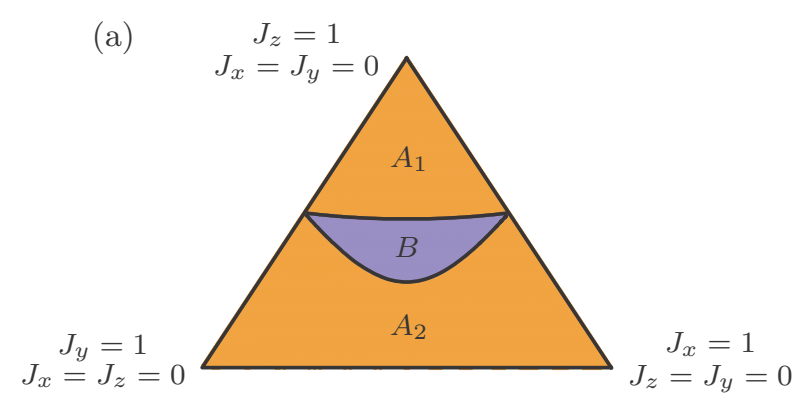

(b)

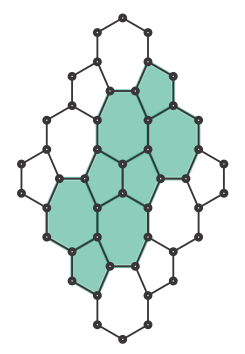

(c)

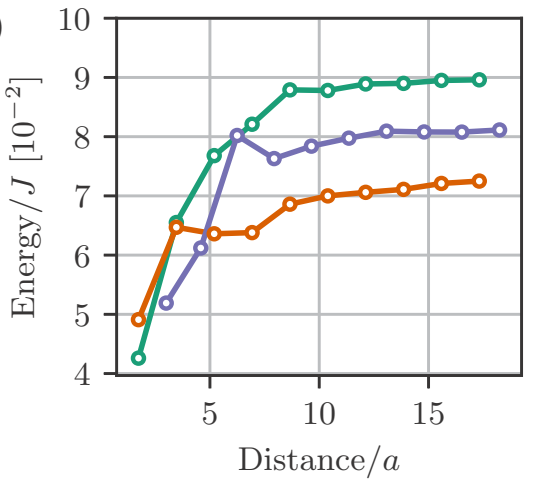

FIG. 2. (a) Phase diagram of the vortex-free sector of Hamiltonian (3). Phases $A_{1}$ and $A_{2}$ are topologically equivalent and realize the Abelian $\mathbb{Z}_{2}$ topological order. Phase $B$ realizes the non-Abelian Ising topological order. (b) Flux configuration of lowest excited state for a system on the torus $L \boldsymbol{e}_{1} \times L \boldsymbol{e}_{2}$ with $L=2$. Colored plaquettes have flux $\pi$. (c) Energy cost per vortex for a pair of vortices as a function of their separation on a torus with $L=10$ and $J_{x}=J_{y}=$ $J_{z}=J$. For the green curve, vortices are in the pentagons. Vortices in heptagons are shown in orange. For the violet curve, one vortex is in a heptagon and the other in a pentagon.

without loss of generality, we find the following: (i) For a cluster of $2 \times 2$ unit cells, the energy computed for all vortex configurations [29] singles out the vortex-free sector with energy -3.1044 per unit cell and an excitation gap of 0.0106 . The first excited sector is a cluster of $\pi$ vortices in half of the plaquettes as displayed in Fig. 2(b). This first excited state is particularly affected by finite-size effects, while we conjecture that the first excited state in larger samples is realized by a single pair of vortices close to each other [29]. (ii) Upon increasing system size, the energy of the vortex-free configuration extrapolates to -3.0971 in the thermodynamic limit. (iii) The energy cost of nucleating a pair of vortices tends to a nonzero constant with increasing separation, indicating that it is not energetically favorable to nucleate isolated vortices [Fig. 2(c)]. Based on this numerical evidence, we consider the vortex-free sector for our subsequent analysis of the pentaheptite Kitaev spectrum.

Phase diagram. The state with all $W_{\text {hep }}=+i, W_{\text {pen }}=-i$ and that with $W_{\text {hep }}=-i, W_{\text {pen }}=+i$ are degenerate and related by time-reversal symmetry. Thus, in the vortex-free sector, the system spontaneously breaks time-reversal symmetry. Without loss of generality, we discuss the phases for $J_{x, y, z}>0$, as the sign of the couplings is irrelevant: A change in the sign of $J_{x}$ or $J_{y}$ can be reabsorbed by changing the sign of an even number of $u_{j k}$ per plaquette without adding vortices. At the same time, $J_{z}<0$ can be mapped to a configuration with

$J_{z}>0$ and an odd number of $u_{j k}$ 's per plaquette with flipped signs. This move adds a vortex in each plaquette, sending each configuration to its time-reversed partner, and does not affect its energy [29].

As shown in the ternary phase diagram Fig. 2(a), we find three gapped phases, which are separated by first-order phase transitions [29] at the phase boundaries given by

$$
J_{z}^{2}=J_{x}^{2} \pm \sqrt{2} J_{x} J_{y}+J_{y}^{2},
$$

where the gap in the Majorna single-particle spectrum closes.

Phases $A_{1}$ and $A_{2}$ are conveniently understood in a limit where one of the couplings $J_{x}, J_{y}$, or $J_{z}$ is much larger than the others. This is a good starting point for a perturbation theory in the Majorana fermion representation [39]. One finds [29] that only noncontractible loops give a flux-dependent correction to the energy. In particular, a loop with $n$ weak bonds of strength $J$ gives a correction of order $J^{n}$. Moreover, loops of odd length do not give any shift in energy as their contribution is canceled by their time-reversal partners. Assume $J_{z} \gg J_{x}, J_{y}$ in $A_{1}$. The first nontrivial correction is given by the loops of length 10 involving adjacent pentagonal and heptagonal plaquettes. The Hamiltonian in sixth-order perturbation theory reads

$$
H_{\text {eff }}^{(6)}=\text { const }-\frac{7}{128}\left(\frac{J_{x}^{4} J_{y}^{2}}{\left|J_{z}\right|^{5}}+\frac{J_{x}^{2} J_{y}^{4}}{\left|J_{z}\right|^{5}}\right) W_{\text {pen }} W_{\text {hep }} .
$$

In $A_{2}$, assume $J_{y} \gg J_{x}, J_{z}$ without loss of generality as the model is symmetric under $J_{x} \leftrightarrow J_{y}$. The first nontrivial contribution arises in fourth-order perturbation theory from the loops of length 8 involving two pentagons. This correction does not provide information on the energy cost of vortices in the heptagonal plaquettes. This enters in sixth-order perturbation theory via the loops of length 10 enclosing a pentagon and a heptagon. The perturbative Hamiltonian up to sixth order for the phase $A_{2}$ is

$$
H_{\text {eff }}^{(6)}=\text { const }+\frac{5 J_{x}^{4}}{16\left|J_{y}\right|^{3}} W_{\text {pen }} W_{\text {pen }^{\prime}}-\frac{7 J_{x}^{2} J_{z}^{4}}{128\left|J_{y}\right|^{5}} W_{\text {pen }} W_{\text {hep }} .
$$

From (6) and (7), we see that both in $A_{1}$ and in $A_{2}$ the vortex sector is gapped and the ground state is in the flux-free sector, i.e., $W_{\text {hep }}=+i$ and $W_{\text {pen }}=-i$. To study the vortex excitations, consider the phase $A_{1}$ in the limit $J_{z} \gg J_{x}, J_{y}$. A pair of vortices can be created in two heptagonal plaquettes or in two pentagonal ones. The energy of these vortices shows little dependence on their separation. On the other hand, a single pair of vortices in a heptagon and a pentagon changes the fermionic parity and it is thus unphysical. These vortices do not carry unpaired Majorana modes. Similar results hold for the $A_{2}$ phase. These observations, together with the fourfold ground-state degeneracy, reproduce the fusion rules and the topological degeneracy of $\mathbb{Z}_{2}$ topological order [6] and support the claim that these phases realize the same topological order as the same limit of Kitaev models on Archimedean lattices.

Phase $B$, which also contains the isotropic point $J_{x}=J_{y}=$ $J_{z}$, is the chiral non-Abelian spin liquid. Our numerical studies suggest that both the vortex sector and the fermionic sector of this phase are gapped [see Supplemental Material and Fig. 2(c)]. Hence, vortices have well-defined statistics. This 
can be entirely determined by the Chern number $C$ associated with the Majorana spectrum according to the 16-fold way for Majorana fermions in a $\mathbb{Z}_{2}$ background gauge field [6]. We find [40] that the spectral gap at half filling has $|C|=1$. An odd Chern number is linked to non-Abelian statistics of the vortex excitation which carries an unpaired Majorana zero mode (MZM). In the presence of well-isolated vortices, these MZMs can be resolved already via exact diagonalization. A pair of MZMs $\left\{\gamma_{i}, \gamma_{j}\right\}$ can be used to construct a nonlocal fermionic degree of freedom $a=1 / 2\left(\gamma_{i}+i \gamma_{j}\right)$ with an associated two-dimensional Fock space, such that a system of isolated $2 n$ vortices possess a topological degeneracy $2^{n}$. Taking into account the noncontractible loops on the torus and imposing fermionic parity conservation, the topological degeneracy for $2 n$ vortices in the $B$ phase is $2^{n+1}$. Therefore, the chiral non-Abelian spin liquid of the $B$ phase is linked to Ising field theory. It is the same phase that can be induced by a magnetic field in Kitaev's model on the honeycomb lattice, albeit in this case at the cost of exact solvability [6]. Here, it is accompanied by a spontaneous breaking of time-reversal symmetry (by choosing all fluxes to be 0 or $\pi)$, as it is the case for the KYK model in Ref. [7]. Other possible realizations of this phase include the $v=5 / 2 \mathrm{FQHE}$ [41] and two-dimensional (2D) topological superconductors [42]. The exact solubility of the model in the $B$ phase offers the opportunity to study its chiral topological edge states for a geometry with open boundary conditions, as presented in Fig. 3(a). The boundary theory of the Ising topological order is a single chiral Majorana fermion mode, in accordance with $|C|=1$.

Coupled wire limit. The limit $J_{z}=J_{x} \gg J_{y}$ is particularly interesting to study the gapped non-Abelian chiral spin-liquid phase. In this limit, (1) can be viewed as a collection of critical one-dimensional Ising chains with alternating $x$ - and $z$-type terms [see Fig. 3(b)] that are weakly coupled with $y$-type terms $[43,44]$. The same limit can be considered for the original Kitaev honeycomb model, which leads to a brick-wall lattice of weakly coupled chains. Thus, by merely changing the geometry of how the chains are connected, one can go from the Abelian Kitaev honeycomb model to a non-Abelian Kitaev model. This represents one of the main advantages of the pentaheptite lattice over the KYK model. In fact, the latter cannot be obtained by a simple coupled wire construction, since in the non-Abelian limit $J^{\prime} \ll J$ (see Ref. [7]), it consists of disconnected triangles. Recent ideas using Majorana-fermion-based "topological hardware" offer a promising route toward realizing topologically ordered spin models (the "topological software") [45-47].

Extensions to 3D. Non-Archimedean lattices in three dimensions are abundant and go beyond the classification studied in Refs. [11,48]. It is interesting to notice how the pentaheptite lattice has a natural 3D extension in a threecoordinated lattice with elementary loops of odd length, e.g., the $(9,3) a$ lattice [11]. As such, it is amenable to an exact solution of the Kitaev model and spontaneously breaks timereversal symmetry, as the pentaheptite lattice in 2D.

The $(9,3) a$ lattice has so far been understood mainly in terms of stacked honeycomb layers via midbond sites. It can, however, alternatively be obtained from the pentaheptite lattice by replacing the bonds shared by a pair of heptagons
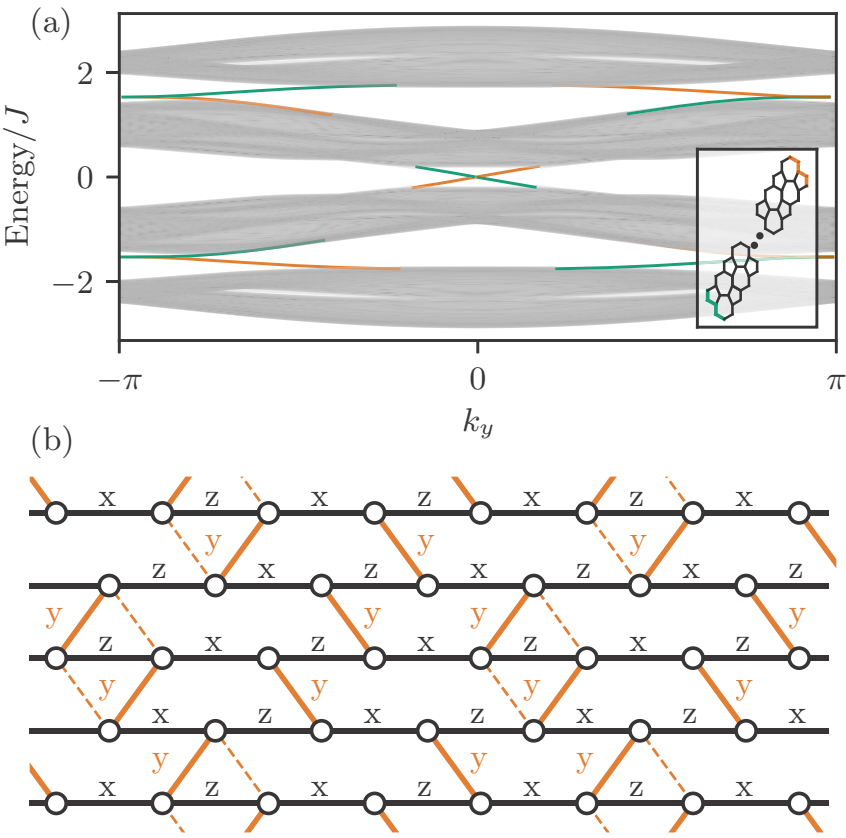

FIG. 3. (a) Boundary spectrum for a ribbon with open boundary conditions along $\boldsymbol{e}_{1}$ and periodic along $\boldsymbol{e}_{2}$, with $J_{x}=J_{y}=J_{z}=J$. The ribbon is composed of 50 unit cells along $\boldsymbol{e}_{1}$. The Chern numbers of the three gaps are $C=-1,+1,-1$ from bottom to top. Modes localized at one edge are plotted in green and those localized at the other edge in orange. The inset shows the edge terminations of the ribbon with respective colors. (b) Non-Abelian chiral spin liquid (phase $B$ ) arising from weakly coupled spin chains. The Kitaev pentaheptite model can be deformed to an array of spin chains with alternating $\sigma^{x}-\sigma^{x}$ and $\sigma^{z}-\sigma^{z}$ coupling that are weakly coupled via $\sigma^{y}-\sigma^{y}$ interactions (orange lines). The dashed lines represent the $\sigma^{y}-\sigma^{y}$ interactions for the coupled wire construction of the honeycomb lattice.

along $\boldsymbol{e}_{1}-\boldsymbol{e}_{2}$ with triangular spirals. The fact that the nonArchimedean 2D lattice studied here can originate from a simpler Archimedean 3D lattice may pave the way to candidate materials for this model which have not been considered previously, and stresses that non-Archimedean systems may generically arise from the dimensional reduction of an Archimedean parent lattice.

Summary. We have generalized the Kitaev spin-liquid paradigm to non-Archimedean lattices, and find the Kitaev pentaheptite model to host an Ising-type non-Abelian chiral spin liquid. Towards a possible realization of this state of matter, we find that the Kitaev honeycomb material $\alpha-\mathrm{RuCl}_{3}$ forms the pentaheptite lattice under uniaxial strain. A future challenge will be to accomplish this experimentally, e.g., by substrate engineering, and, from a complementary theoretical point of view, to provide a microscopic derivation of the magnetic exchange interactions in the modified pentaheptite structure. More broadly, while there has been some systematic work on frustrated magnetism on Archimedean lattices [49], a comparable effort on both Lieb-type and non-Archimedean lattices is still lacking, and we hope that our work will motivate such studies.

Acknowledgments. We thank Oleg Tchernyshyov for helpful comments and discussions. V.P. acknowledges support 
from the European Research Council under Grant Agreement No. 771503 and NCCR MaNEP of the Swiss National Science Foundation. S.O. was supported by the Swiss National Science Foundation under Grant No. 200021_169061. T.N. and S.S.T. acknowledge support from the European Unions Horizon 2020 research and innovation program (ERC-StGNeupert-757867-PARATOP). G.B. acknowledges the support of the Wilhelm Wien Institute as a distinguished Wilhelm Wien professor at the University of Würzburg, where this work was initiated, DST-SERB (India) for a SERB
Distinguished Fellowship, and research at Perimeter Institute, as a DVRC, is supported by the Government of Canada through Industry Canada and by the Province of Ontario through the Ministry of Research and Innovation. The work in Würzburg and Dresden is funded by the Deutsche Forschungsgemeinschaft (DFG, German Research Foundation) through Project-ID 258499086 - SFB 1170, Project-ID 247310070 - SFB 1143, and through the Würzburg-Dresden Cluster of Excellence on Complexity and Topology in Quantum Matter-ct.qmat Project-ID 39085490 - EXC 2147.
[1] P. Fazekas and P. W. Anderson, Philos. Mag. 30, 423 (1974).

[2] P. W. Anderson, Science 235, 1196 (1987).

[3] V. Kalmeyer and R. B. Laughlin, Phys. Rev. Lett. 59, 2095 (1987).

[4] A. Kitaev, Ann. Phys. 303, 2 (2003).

[5] R. Moessner and S. L. Sondhi, Phys. Rev. Lett. 86, 1881 (2001).

[6] A. Kitaev, Ann. Phys. 321, 2 (2006).

[7] H. Yao and S. A. Kivelson, Phys. Rev. Lett. 99, 247203 (2007).

[8] J. Fu, Phys. Rev. B 100, 195131 (2019).

[9] M. Barkeshli, H.-C. Jiang, R. Thomale, and X.-L. Qi, Phys. Rev. Lett. 114, 026401 (2015).

[10] M. Hermanns, K. O’Brien, and S. Trebst, Phys. Rev. Lett. 114, 157202 (2015).

[11] K. O'Brien, M. Hermanns, and S. Trebst, Phys. Rev. B 93, 085101 (2016).

[12] J.-J. Miao, H.-K. Jin, F.-C. Zhang, and Y. Zhou, arXiv:1806.10960.

[13] M. Greiter and R. Thomale, Phys. Rev. Lett. 102, 207203 (2009).

[14] M. Greiter, D. F. Schroeter, and R. Thomale, Phys. Rev. B 89, 165125 (2014).

[15] T. Meng, T. Neupert, M. Greiter, and R. Thomale, Phys. Rev. B 91, 241106(R) (2015).

[16] P. Lecheminant and A. M. Tsvelik, Phys. Rev. B 95, 140406(R) (2017).

[17] J.-Y. Chen, L. Vanderstraeten, S. Capponi, and D. Poilblanc, Phys. Rev. B 98, 184409 (2018).

[18] B. Scharfenberger, R. Thomale, and M. Greiter, Phys. Rev. B 84, 140404(R) (2011).

[19] O. I. Motrunich, Phys. Rev. B 72, 045105 (2005).

[20] J. Knolle, D. L. Kovrizhin, J. T. Chalker, and R. Moessner, Phys. Rev. Lett. 112, 207203 (2014).

[21] G. Jackeli and G. Khaliullin, Phys. Rev. Lett. 102, 017205 (2009)

[22] Y. Singh, S. Manni, J. Reuther, T. Berlijn, R. Thomale, W. $\mathrm{Ku}, \mathrm{S}$. Trebst, and P. Gegenwart, Phys. Rev. Lett. 108, 127203 (2012).

[23] K. W. Plumb, J. P. Clancy, L. J. Sandilands, V. V. Shankar, Y. F. Hu, K. S. Burch, H.-Y. Kee, and Y.-J. Kim, Phys. Rev. B 90, 041112(R) (2014).

[24] M. Hermanns, I. Kimchi, and J. Knolle, Annu. Rev. Condens. Matter Phys. 9, 17 (2018).
[25] S. Trebst, arXiv:1701.07056.

[26] E. H. Lieb, Phys. Rev. Lett. 62, 1201 (1989).

[27] V. H. Crespi, L. X. Benedict, M. L. Cohen, and S. G. Louie, Phys. Rev. B 53, R13303(R) (1996).

[28] M. Deza, P. W. Fowler, M. Shtogrin, and K. Vietze, J. Chem. Inf. Comput. Sci. 40, 1325 (2000).

[29] See Supplemental Material at http://link.aps.org/supplemental/ 10.1103/PhysRevB.101.041114 for details on the DFT calculations and a detailed analysis of the model phases.

[30] G. Kresse and J. Hafner, Phys. Rev. B 48, 13115 (1993).

[31] G. Kresse and J. Furthmüller, Comput. Mater. Sci. 6, 15 (1996).

[32] P. E. Blöchl, Phys. Rev. B 50, 17953 (1994).

[33] G. Kresse and D. Joubert, Phys. Rev. B 59, 1758 (1999).

[34] J. P. Perdew, K. Burke, and M. Ernzerhof, Phys. Rev. Lett. 77, 3865 (1996).

[35] S. Biswas, Y. Li, S. M. Winter, J. Knolle, and R. Valenti, Phys. Rev. Lett. 123, 237201 (2019).

[36] F. L. Pedrocchi, S. Chesi, and D. Loss, Phys. Rev. B 84, 165414 (2011).

[37] E. H. Lieb, Phys. Rev. Lett. 73, 2158 (1994).

[38] S. Chesi, A. Jaffe, D. Loss, and F. L. Pedrocchi, J. Math. Phys. 54, 112203 (2013).

[39] O. Petrova, P. Mellado, and O. Tchernyshyov, Phys. Rev. B 90 , 134404 (2014).

[40] T. Fukui, Y. Hatsugai, and H. Suzuki, J. Phys. Soc. Jpn. 74, 1674 (2005).

[41] G. Moore and N. Read, Nucl. Phys. B 360, 362 (1991).

[42] D. A. Ivanov, Phys. Rev. Lett. 86, 268 (2001).

[43] P.-H. Huang, J.-H. Chen, P. R. S. Gomes, T. Neupert, C. Chamon, and C. Mudry, Phys. Rev. B 93, 205123 (2016).

[44] P.-H. Huang, J.-H. Chen, A. E. Feiguin, C. Chamon, and C. Mudry, Phys. Rev. B 95, 144413 (2017).

[45] E. Sagi, H. Ebisu, Y. Tanaka, A. Stern, and Y. Oreg, Phys. Rev. B 99, 075107 (2019).

[46] L. A. Landau, S. Plugge, E. Sela, A. Altland, S. M. Albrecht, and R. Egger, Phys. Rev. Lett. 116, 050501 (2016).

[47] S. Plugge, L. A. Landau, E. Sela, A. Altland, K. Flensberg, and R. Egger, Phys. Rev. B 94, 174514 (2016).

[48] A. F. Wells, Three-Dimensional Nets and Polyhedra, Wiley monographs in crystallography (Wiley, New York, 1977).

[49] D. J. J. Farnell, O. Götze, J. Richter, R. F. Bishop, and P. H. Y. Li, Phys. Rev. B 89, 184407 (2014). 